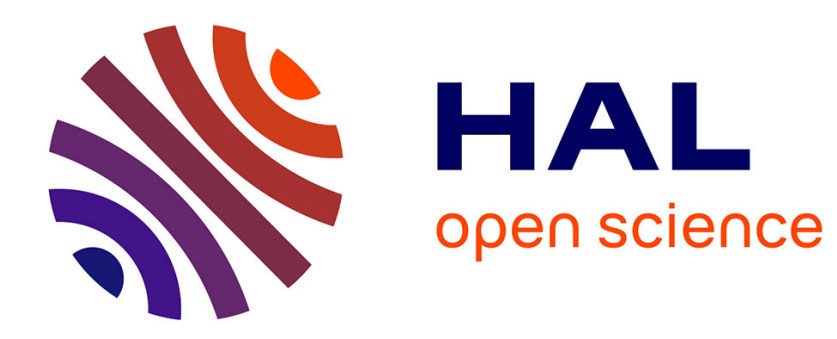

\title{
Les systématiciens à l'épreuve du barcoding
}

\author{
Isabelle Mauz, Elsa Faugere
}

\section{To cite this version:}

Isabelle Mauz, Elsa Faugere. Les systématiciens à l'épreuve du barcoding. Revue d'Anthropologie des Connaissances, 2013, 7, 2 (2), pp.433-459. 10.3917/rac.019.0433 . halshs-01664808

\section{HAL Id: halshs-01664808 https://shs.hal.science/halshs-01664808}

Submitted on 15 Dec 2017

HAL is a multi-disciplinary open access archive for the deposit and dissemination of scientific research documents, whether they are published or not. The documents may come from teaching and research institutions in France or abroad, or from public or private research centers.
L'archive ouverte pluridisciplinaire HAL, est destinée au dépôt et à la diffusion de documents scientifiques de niveau recherche, publiés ou non, émanant des établissements d'enseignement et de recherche français ou étrangers, des laboratoires publics ou privés. 


\section{LES SYSTÉMATICIENS À L'ÉPREUVE DU BARCODING \\ Une étude des pratiques d'enrôlement scientifique \\ Isabelle Mauz, Elsa Faugère}

\section{S.A.C. | « Revue d'anthropologie des connaissances »}

2013/2 Vol. 7, nº 2 | pages 433 à 459

\section{Article disponible en ligne à l'adresse :}

https://www.cairn.info/revue-anthropologie-des-connaissances-2013-2-page-433.htm

\section{Pour citer cet article :}

Isabelle Mauz, Elsa Faugère« Les systématiciens à l'épreuve du barcoding. Une étude des pratiques d'enrôlement scientifique », Revue d'anthropologie des connaissances 2013/2 (Vol. 7, n² 2), p. 433-459.

DOI 10.3917/rac.019.0433

Distribution électronique Cairn.info pour S.A.C..

(C) S.A.C.. Tous droits réservés pour tous pays.

La reproduction ou représentation de cet article, notamment par photocopie, n'est autorisée que dans les limites des conditions générales d'utilisation du site ou, le cas échéant, des conditions générales de la licence souscrite par votre établissement. Toute autre reproduction ou représentation, en tout ou partie, sous quelque forme et de quelque manière que ce soit, est interdite sauf accord préalable et écrit de l'éditeur, en dehors des cas prévus par la législation en vigueur en France. Il est précisé que son stockage dans une base de données est également interdit. 


\section{LES SYSTÉMATICIENS À L'ÉPREUVE DU BARCODING \\ Une étude des pratiques d'enrôlement scientifique}

ISABELLE MAUZ

ELSA FAUGÈRE

\section{RÉSUMÉ}

Dans un contexte qualifié de $6^{\mathrm{e}}$ crise d'extinction des espèces, les inventaires de la biodiversité ont acquis une valeur et une urgence inédites. Les scientifiques - qu'on appelle taxonomistes ou systématiciens - dont le métier consiste à explorer, inventorier et collecter les espèces vivantes se sont saisis de nouveaux outils pour accélérer et améliorer leur entreprise d'inventaire de la biodiversité. Depuis la parution en 2003 d'un article fondateur de Hebert et al., l'un d'eux occupe une place tout à fait centrale : le barcoding. Inventé et promu par une partie des systématiciens (les molécularistes), sa réussite exige la collaboration active des systématiciens qui fondent leur travail sur l'observation des caractères morphologiques (les morphologistes). En nous appuyant sur des enquêtes menées dans des terrains contrastés, nous analysons comment les molécularistes s'efforcent d'enrôler les morphologistes dans le barcoding. Nous montrons que les molécularistes ont une stratégie d'enrôlement plurielle, qui repose sur un ensemble de compétences pédagogiques, rhétoriques et sociales et leur permet de tenir compte de la grande diversité et singularité des morphologistes et des conditions d'exercice de leur pratique. Nous mettons également en évidence des facteurs qui favorisent cette stratégie d'enrôlement, comme la participation physique des molécularistes aux campagnes de 
collection et le soutien de morphologistes convertis au barcoding. Cet article entend ainsi contribuer à la connaissance et à l'analyse des pratiques d'enrôlement scientifique, restées relativement peu explorées.

Mots clés : enrôlement, collaboration, systématique, barcoding, molécularistes, morphologistes, inventaire, biodiversité

\section{INTRODUCTION}

Au début des années 2000, un article publié dans les Proceedings of the Royal Society de Londres allait connaître un retentissement considérable dans la communauté scientifique des biologistes intéressés par la découverte et l'identification d'espèces nouvelles, que ce soit dans une perspective taxonomique ou évolutionniste. II s'agit de l'article du généticien canadien Paul N. Hebert et de trois de ses collègues de l'université de Guelph au Canada : Alina Cywinska, Shelley L. Ball et Jeremy R. de Waard. Intitulé "Biological Identification through DNA barcodes », cet article a initié une série d'initiatives internationales en matière d'inventaires de la biodiversité à l'échelle mondiale et contribué à modifier de manière significative l'exercice même du métier de systématicien, comme nous le verrons par la suite. II a notamment conduit à la création, en 2004, du Consortium for the Barcode of Life, initiative internationale, financée par une fondation américaine, qui réunit plus de 200 organisations de 50 pays différents - dont le Muséum National d'Histoire Naturelle de Paris - autour d'un même objectif : faire du barcoding un modèle global (a global standard) pour l'identification des espèces biologiques (http://www.barcodeoflife.org/content/about/what-cbol). En faisant du barcoding une technique présentée comme révolutionnaire pour identifier et séparer les espèces vivantes, l'article d'Herbert et al. a sans aucun doute contribué à bousculer les frontières disciplinaires internes aux différents champs de la biologie contemporaine, en poussant différents groupes de systématiciens à collaborer autour de mêmes projets d'inventaires et de collectes d'une biodiversité considérée comme menacée. Sans être nouvelles, les collaborations dans les sciences de la vie ont acquis au cours des dernières décennies une ampleur sans précédent (Parker et al., 2010). Elles se nouent non seulement entre des producteurs de connaissances qui se ressemblent et forment des collectifs relativement homogènes mais aussi entre des catégories de personnes que l'on avait tendance à opposer. D'une part, les collectifs de travail peuvent regrouper des chercheurs ayant différentes manières de connaître le vivant (Pickstone, 2000). Les historiens des sciences ont ainsi contesté le récit classique selon lequel les sciences expérimentales et d'analyse auraient évincé l'histoire naturelle. Kohler (2002) a mis en évidence l'existence d'espaces frontières (border zones) entre les naturalistes de terrain et les scientifiques 
de laboratoire. Strasser (20I I, 20I2) a souligné l'importance des pratiques de mise en collection dans le développement des sciences de laboratoire comme la biologie moléculaire. En se centrant sur les pratiques de collectes et de mise en collection, leurs travaux ont permis de donner une autre vision de l'histoire des sciences, montrant l'importance persistante de l'histoire naturelle dans les sciences de la vie tout au long du vingtième siècle et l'émergence de «cultures hybrides ॥. D'autre part, des collaborations s'établissent entre des chercheurs et des non-chercheurs, qui peuvent être des gestionnaires de la nature (Mauz, Granjou, 2013) ou des naturalistes amateurs (Lawrence, 2006 ; Waterton, Ellis, 2006 ; Charvolin, Micoud, Nyhart, 2007). Là encore, le phénomène n'est assurément pas nouveau ni même récent mais il tend à se développer. Par exemple, l'essor des technologies de la communication a amplifié et réactualisé la contribution des botanistes amateurs à la production de connaissances dans cette discipline (Heaton et al., 20II). De plus en plus, les collectifs de travail dans les sciences de la vie rassemblent ainsi des personnes qui ont des manières de connaître et des statuts très variés.

Les travaux sur les collaborations ont largement éclairé les motivations et les réticences des divers producteurs de connaissances à collaborer (cf. Parker et al., 2010). Plusieurs articles ont notamment documenté les réserves que peuvent avoir les amateurs à livrer des données auxquelles ils sont extrêmement attachés. On sait en revanche relativement peu de choses sur la façon dont les chercheurs s'y prennent pour initier des collaborations, les pratiques qu'ils mettent en œuvre pour enrôler (Callon, 1986) d'autres producteurs de connaissances dans leur activité, les compétences sociales qu'ils mobilisent et les facteurs qui favorisent leur entreprise d'enrôlement. En bref, si on sait mieux, désormais, que les chercheurs collaborent entre eux et avec d'autres et pourquoi ils le font (ou pas), on ignore encore largement ce qu'ils font pour y parvenir. Cet article souhaite contribuer à combler cette lacune, à partir du cas de l'introduction dans une discipline ancienne, la systématique, d'une nouvelle technique d'analyse moléculaire : le barcoding. Les enquêtes que nous avons menées dans des terrains contrastés permettent de saisir le travail concret déployé par les systématiciens promoteurs du barcoding pour convaincre d'autres systématiciens de contribuer à leur entreprise ainsi que les circonstances qui favorisent ou compliquent ce travail.

Le texte comporte quarte parties. La première présente le barcoding et notre méthode d'enquête. Nous montrons que le barcoding a creusé la distinction entre deux groupes de systématiciens, les molécularistes, qui maîtrisent et promeuvent cette nouvelle technologie, et les morphologistes, tout en nécessitant la collaboration de ces derniers. La deuxième expose les exigences des molécularistes à l'égard des morphologistes, qui doivent collecter, préparer et équiper les spécimens d'une certaine manière pour que le barcoding puisse avoir lieu. La troisième porte sur les pratiques que développent les molécularistes pour enrôler les morphologistes. Enfin, la quatrième partie met en avant les circonstances qui favorisent ou au contraire compliquent la réussite de ces pratiques d'enrôlement. 


\section{CAS D’ÉTUDE ET MÉTHODE D’ENQUÊTE}

\section{L’arrivée du barcoding dans la systématique}

La systématique est une discipline scientifique très ancienne qui a pour but d'identifier et de classer les espèces. Les systématiciens se sont longtemps fondés sur la seule observation de critères morphologiques pour accomplir leur travail. Au cours du vingtième siècle, certains systématiciens ont eu l'idée de recourir à une analyse moléculaire (d'abord de protéines, puis de l'ADN) pour identifier et classer les espèces. II y a eu dès lors des systématiciens morphologistes et des systématiciens molécularistes, qui ont des manières différentes de connaître le vivant (l'histoire naturelle pour les morphologistes, l'analyse pour les molécularistes') et constituent des groupes en bonne partie distincts. Si un morphologiste peut en effet comprendre les principes de travail des molécularistes et inversement, il est en revanche difficile d'être à la fois très bon morphologiste et très bon moléculariste, étant donné le temps de formation nécessaire pour parvenir au meilleur niveau et la nécessité d'une pratique intensive pour s'y maintenir. La plupart des morphologistes ne deviennent donc pas des molécularistes même si certains d'entre eux sont allés se former aux techniques moléculaires aux États-Unis et symétriquement, les molécularistes n'apprennent pas à reconnaître les espèces sur des bases morphologiques, sauf dans le cas de quelques espèces dont ils sont spécialistes.

Cependant, jusqu'à une période récente, l'observation des critères morphologiques l'a emporté sur l'analyse moléculaire. La situation a changé au début des années 2000, avec l'invention d'une nouvelle technique d'analyse moléculaire, le barcoding, que ses promoteurs ont présentée comme révolutionnaire et qui s'est rapidement diffusée. Le barcoding repose sur la possibilité d'utiliser une séquence d'un gène mitochondrial, le gène $\mathrm{CO}$, comme outil de diagnostic d'un très grand nombre d'espèces animales. II nécessite des analyses poussées en laboratoire, requérant une instrumentation coûteuse et sophistiquée (thermocycleurs pour amplifier l'ADN, séquenceurs, etc.). Une base de données (nommée BOLD pour Barcoding of Life Database) rassemblant les barcodes est en cours de constitution à l'université de Guelph (Canada), où le barcoding a été inventé. Le barcoding est donc une technique standardisée, puisque la même séquence de gène est analysée et archivée selon la même

\footnotetext{
I Sur les manières de connaître (ways of knowing), voir Pickstone (2000). Pickstone propose de distinguer trois grandes manières de connaître, caractérisées par des questions spécifiques ainsi que par des pratiques matérielles et cognitives : « ... natural history - the describing and classification of things; [...] analysing into various kinds of elements, like germ layers, cells and chemical elements; and [...] experimenting to control phenomena and to systematically create novelties » (Pickstone, 2000 : 2, souligné dans le texte). Pour une discussion de cette notion, voir Strasser et de Chadarevian (20I I).
} 
procédure, et globalisée puisque tous les barcodes produits sont censés être centralisés dans une base de données unique.

Les molécularistes ont inventé le barcoding mais ils ne sont pas en mesure, à eux seuls, d'assurer sa réussite : si ce sont eux qui extraient et analysent l'ADN et alimentent la base de données BOLD, ils ont impérativement besoin que le plus grand nombre d'espèces possibles soient collectées et identifiées par des morphologistes pour que chaque barcode puisse être mis en regard d'une espèce dûment identifiée et puisse ensuite servir de référence. La base de données globale de Guelph ne peut donc se constituer sans la participation active d'une multitude de morphologistes.

Le barcoding a fait l'objet d'un certain nombre de travaux par des philosophes et des sociologues des sciences (Dupré, 2006 ; Larson, 2007 ; Ellis, 2008 ; Ellis, Waterton, Wynne, 2009 ; Waterton, 2010). En particulier, Ellis, Waterton et Wynne ont conduit une enquête approfondie dans des institutions ayant joué un rôle clef dans le développement de ce nouveau champ techno-scientifique au Royaume-Uni, au Canada et aux États-Unis et ont analysé certains de ses enjeux et effets socio-politiques. Ces auteurs ont en particulier discuté la question des rapports entre le barcoding, ses publics et la démocratie, en se focalisant sur le public général imaginé et forgé par le barcoding. Le barcoding a en effet été présenté par ses promoteurs comme un instrument de démocratisation de la systématique (Dupré, 2006 ; Larson, 2007 ; Ellis, Waterton, Wynne, 2009). L'affirmation qu'il permettra à tout un chacun partout dans le monde de « lire » la diversité spécifique du monde naturel et, par suite, de l'apprécier et de la préserver constitue l'une des principales légitimations du barcoding comme outil d'identification des espèces. C'est notamment sur cette promesse que s'appuient les promoteurs du barcoding pour parler de technique révolutionnaire. La systématique deviendrait à terme accessible et utile à un public général actuellement « bio-illettré » qu'il importe de convertir à la nécessité de préserver la biodiversité. Étudiant les caractéristiques de ce public général globalisé fictif que les promoteurs du barcoding doivent imaginer pour justifier le développement de leur innovation, Ellis, Waterton et Wynne soulignent l'opération d'homogénéisation et d'occultation des différences des rapports à la nature qui sous-tend sa production.

Les articles cités ont en revanche peu approfondi le cas des systématiciens morphologistes. C'est pour notre part ce « premier public » (Ellis, Waterton, Wynne, 2009), bien réel, qui a retenu notre attention. Nous chercherons à comprendre comment les molécularistes s'y prennent pour enrôler les morphologistes dans le barcoding.

\section{Terrains et méthode d'enquête}

Nous avons observé la façon dont les molécularistes tentent d'enrôler des morphologistes dans le barcoding à l'occasion d'enquêtes sur des inventaires de la biodiversité dans des terrains contrastés : les modules marins des expéditions 
naturalistes d'une part, l'inventaire biologique généralisé du Mercantour² d'autre part.

Notre première enquête sur les modules marins des expéditions naturalistes s'est déroulée sur l'île de Santo, en octobre 2006. Organisée au Vanuatu par le Muséum national d'histoire naturelle (MNHN), l'ONG Pro-Natura International et l'Institut de Recherche pour le Développement, Santo a été présenté par les médias de l'époque comme "la plus grande expédition naturaliste de tous les temps ॥ (Faugère, 2008). Ses installations comportaient un « atelier barcode $\|^{3}$, situé dans le laboratoire de plein air aménagé dans le grand hangar du Maritime College de l'île de Santo. L'atelier se distinguait des autres postes de travail par son instrumentation plus sophistiquée ainsi que par la vivacité des discussions et des tensions entre les barcodeurs et les morphologistes. Les premiers reprochaient aux seconds d'être plus intéressés par l'accumulation de spécimens et d'espèces «nouvelles pour la science » que par la résolution de questionnements scientifiques et voyaient en eux de simples « collectionneurs de timbres-poste ». Symétriquement, les morphologistes trouvaient les barcodeurs dénués de véritable intérêt et curiosité naturalistes et soulignaient leur incapacité à reconnaître les espèces selon des critères morphologiques et leur dépendance aux « vrais » systématiciens.

Nous avons effectué une deuxième enquête sur les modules marins des expéditions naturalistes en mai 2010, à l'extrême sud de Madagascar, lors d'une nouvelle expédition baptisée Atimo Vatae. Là aussi, les barcodeurs étaient présents et avaient leur atelier. Mais ils s'étaient apparemment réconciliés avec les morphologistes et les querelles autour du barcoding ne semblaient plus qu'un lointain souvenir. Tous parlaient désormais de « systématique intégrative » et soulignaient la nécessité d'une collaboration étroite entre les morphologistes et les barcodeurs. Ces deux enquêtes nous ont permis d'observer les pratiques de collecte et de tri des systématiciens, d'avoir des discussions informelles et de mener des entretiens auprès des participants de l'ensemble des modules marins et de l'atelier barcode.

De 2010 à 2012, nous avons par ailleurs étudié l'inventaire de la biodiversité effectué dans le parc national du Mercantour. Il s'agissait là aussi d'effectuer un inventaire massif de la biodiversité spécifique, cette fois en milieu terrestre. Cet inventaire a été étalé sur plusieurs années (2006-2012) et a concerné la totalité de l'espace du parc national, ce qui a rendu impossible l'organisation d'un atelier barcode sur le terrain. Les morphologistes ont donc dû prendre en charge une partie des tâches nécessitées par le barcoding. Par crainte de les dissuader d'emblée de s'impliquer, le barcoding n’a été introduit que mi-2009, soit trois ans et demi après le démarrage de l'inventaire. L'organisation de ce

2 L'inventaire a en réalité été effectué dans le parc national du Mercantour et le parc naturel voisin des Alpi Marittime, en Italie. Pour des raisons pratiques, l'enquête n'a concerné que le versant français de l'inventaire.

3 Contrairement à ce que suggère l'appellation, seuls la préparation et l'équipement des spécimens pour le barcoding ont été effectués dans l'« atelier barcode ». Les autres étapes du barcoding (extraction, amplification et séquençage de l'ADN) se déroulent au service de systématique moléculaire du MNHN pour les deux premières et au génoscope d'Évry pour la troisième. 
dernier a ainsi fortement différé de celle des modules marins des expéditions naturalistes, en particulier en ce qui concerne le barcoding. Nous avons mené une vingtaine d'entretiens semi-directifs auprès d'agents du parc national du Mercantour et de systématiciens impliqués dans l'inventaire. Deux entretiens ont été réalisés au service de systématique moléculaire du Muséum, l'un avec son directeur, l'autre avec la personne recrutée pour organiser le volet barcode de l'inventaire. Ces entretiens ont été accompagnés d'une visite du service. Le barcoding et ses implications pratiques ont été abordés avec la plupart des autres enquêtés.

Nous avons complété ces enquêtes de terrain par des entretiens avec un jeune barcodeur, Nicolas. Né en 198I, il est suffisamment jeune pour être entré en recherche après l'apparition du barcoding. II ne fait donc pas partie des promoteurs et des initiateurs de cette innovation qui est, pour lui, une technologie « normale », d'emblée au cœur de son travail de recherche. Nous l'avons rencontré pour la première fois en octobre 2006 lors de l'expédition Santo. Il y était chargé de "l'atelier barcode », aux côtés d'un jeune chercheur américain de Guam, Jason, spécialiste du venin des cônes, et de deux chercheurs seniors anatomistes, Ellen, du Smithsonian Institute de Washington et Yuri, de l'Académie des Sciences de Russie. Nous avons eu ensuite deux entretiens avec lui, l'un en mai 2010, à Madagascar, l'autre en juin 2012, au laboratoire de malacologie du MNHN.

\section{Parcours d'un jeune barcodeur}

Nicolas se présente comme un systématicien moléculaire évolutionniste. Systématicien car il s'intéresse à la classification des espèces. Moléculaire car sa contribution à l'entreprise de classification s'appuie sur l'analyse de molécules et plus précisément de l'ADN plutôt que sur l'observation de caractères morphologiques. Évolutionniste car il place ses travaux de recherche dans le contexte de la théorie de l'évolution.

Son parcours témoigne de la difficulté extrême à obtenir un poste en systématique, même en maîtrisant parfaitement les techniques moléculaires. II a soutenu en 2008 une thèse en systématique évolutive, intitulée "Taxonomie intégrative des Turridae : phylogénie, délimitation d'espèces et barcoding ॥. II a ensuite effectué un post-doctorat à l'université de l'Utah puis un autre au CNRS, avant d'être assistant de recherche au MNHN. II a mené jusqu'en janvier 2012 un quatrième post-doctorat, en Suisse, dans un laboratoire privé spécialisé dans l'analyse moléculaire des toxines animales. II a alors commencé un cinquième post-doctorat, dans le cadre d'un projet de recherche financé par l'ANR et coordonné par le directeur du laboratoire de malacologie qui a codirigé sa thèse. En juin 2012, il prévoyait de commencer en septembre un contrat d'ATER dans le domaine de la modélisation, afin d'acquérir de nouvelles compétences et d'augmenter ses chances d'obtenir un poste. II avait alors 29 articles publiés ou sous presse, principalement dans des revues internationales de 
biologie ou d'écologie moléculaire, à haut facteur d'impact, et de malacologie, à moindre facteur d'impact. II postulait simultanément à deux postes en systématique, l'un au laboratoire de malacologie du MNHN, l'autre au Centre de Biologie et de Gestion des Populations (CBGP), à Montpellier.

Sa candidature au MNHN a été retenue et il a pris son poste en janvier 2013.

Les enquêtes réalisées montrent un différentiel de réussite des dispositifs d'enrôlement des systématiciens dans le barcoding selon les terrains. Qu'ils soient amateurs ou professionnels, les participants au module marin des expéditions semblent avoir été rapidement et facilement enrôlés dans le barcoding. Ces entreprises de collecte ont certes dû se réorganiser légèrement avec l'apparition d'un " atelier barcode » et d'une équipe consacrée à la préparation et au conditionnement des spécimens mais le barcoding n'a pas suscité de polémiques de long terme, ni de rejet particulier. L'enrôlement des systématiciens de terrain dans le volet barcoding de l'inventaire de la biodiversité du Mercantour a en revanche été plus laborieux. Malgré les démarches et les efforts des personnes chargées de promouvoir et d'organiser ce volet barcoding, une partie seulement des morphologistes ont été prêts à contribuer à l'entreprise. Comprendre ces différences suppose au préalable de saisir les exigences des molécularistes à l'égard des morphologistes et la stratégie d'enrôlement qu'ils mettent en œuvre.

\section{LES EXIGENCES DES MOLÉCULARISTES À L'ÉGARD DES MORPHOLOGISTES}

Les morphologistes sont à la fois des collecteurs, des conservateurs et des collectionneurs des spécimens qu'ils dénichent sur le terrain (Kohler, 2006 : 433 ; Strasser, $2012: 21$ ). Comme il ne peut y avoir d'activité naturaliste sans constitution de données et d'inscriptions (Strasser, 20I2), saisir des données fait également partie de leurs activités classiques. Les barcodeurs ne demandent pas aux morphologistes d'autres pratiques que celles qu'ils maîtrisent déjà. En revanche, ils leur demandent de les modifier. Pour pouvoir travailler, les barcodeurs ont en effet besoin que les spécimens aient été collectés et préparés d'une certaine manière et qu'ils soient équipés ${ }^{4}$ de données standardisées. Bien qu'il arrive en bout de chaîne de traitement des spécimens, le barcoding influence donc le travail des morphologistes dès ses premières étapes.

L'ADN est une molécule fragile qui se dégrade rapidement. Pour assurer sa protection, les spécimens et les tissus destinés au barcoding doivent être conservés dans de l'alcool très concentré. L'alcool est le produit classiquement

4 Sur la notion d'équipement, voir Vinck (20II). 
employé pour conserver les animaux marins; les morphologistes des expéditions marines n'ont donc pas eu à changer de conservateur. Éventuellement, les molécularistes leur ont simplement demandé de passer à un alcool plus concentré. En revanche, certaines méthodes de piégeage utilisées en entomologie dans l'inventaire de la biodiversité du Mercantour privilégiaient d'autres conservateurs, comme les antigels, qui se sont révélés incompatibles avec le barcoding parce qu'ils dégradent l'ADN. Les molécularistes ont alors dû demander aux morphologistes d'adopter des techniques de piégeage moins satisfaisantes pour ces derniers :

« II y a un type de piégeage, qui est utilisé en entomologie et qui me faisait peur, et à juste raison. C'est ce qu'ils appellent les pièges d'interception. Ils mettent des espèces de bâches dans des voies où circulent les insectes et les insectes viennent se taper sur la bâche et ils tombent et en fait, ils sont récoltés dans de l'antigel. L'antigel conserve bien les spécimens : ils ne pourrissent pas, ils ne se déshydratent pas trop, ils sont ensuite facilement manipulables et donc pour tout ce qui est morphologie, c'est super. Mais par contre pour l'ADN, c'est niet. On a essayé sur un lot de spécimens, on n'a absolument rien eu. II semblerait que ça dépende des antigels, je crois qu'il y a de l'éthylène glycol et du polypropylène glycol. Suivant les antigels, je ne sais plus lequel des deux, enfin il y en a un des deux qui semblerait ne pas être aussi mauvais pour l'ADN. Enfin, celui qu'on a essayé, en tout cas, ça a tout détruit, on n'a eu aucune séquence, enfin, on n'a eu aucun ADN, on n'a rien pu amplifier du tout » (responsable du volet barcoding de l'inventaire de la biodiversité du Mercantour, mai 20I I).

Les morphologistes doivent aussi apprendre à prélever un morceau de tissu, ou une petite partie de l'animal, dont l'ADN est ensuite extrait. Cette opération peut s'avérer passablement compliquée. II faut veiller à bien préserver les tissus, en les imbibant d'alcool suffisamment concentré. Si le spécimen est endommagé par l'opération, des spécimens intacts collectés au même endroit et appartenant selon toute vraisemblance à la même espèce doivent accompagner les tissus prélevés, afin de permettre une identification morphologique de l'animal. En outre, le morceau de tissu prélevé ne doit pas être contaminé par l'ADN d'autres organismes, ce qui amène à exclure certains organes.

Les morphologistes doivent encore équiper les spécimens collectés d'un identifiant qui les suivra constamment, eux et leurs avatars ${ }^{5}$. Un prélèvement sans identifiant ne présente aucun intérêt et est éliminé (" ce n'est plus qu'un bout de chair ", dit un enquêté). Un des acquis de la sociologie des sciences concerne l'importance des inscriptions et des « mobiles immuables », ces objets qui voyagent depuis les lieux où ils ont été collectés jusqu'à un « centre de calcul » où ils peuvent être étudiés tout à loisir (Latour, 1989 : 545). À l'arrivée, les objets en question peuvent être sensiblement différents de ce qu'ils étaient au départ mais un lien solide a été établi entre leurs états successifs, si bien que l'on peut, du moins en théorie, remonter toute la chaîne du point final au

5 Pour une étude plus approfondie sur ce point, voir Granjou et Mauz (2009). 
point initial. Les fragments de spécimen destinés au barcoding font typiquement partie de ces mobiles immuables. Collectés n'importe où dans le monde, ils voyagent jusqu'à des centres de calcul intermédiaires comme le MNHN ou le CBGP puis jusqu'au centre de calcul ultime qu'est BOLD, la base de données de référence globale de l'université de Guelph. Pour les barcodeurs, la difficulté consiste à maintenir le lien entre les barcodes produits et les spécimens dont ils sont issus, de manière à pouvoir remonter des premiers aux seconds. La question de la traçabilité est donc cruciale pour l'entreprise dans son ensemble (Puillandre et al., 2012).

Enfin, les morphologistes doivent saisir un ensemble de données sur les spécimens collectés : dans le Mercantour, ils ont reçu une feuille de données Excel, qu'ils devaient renvoyer au Muséum en même temps que le spécimen à barcoder. Cette feuille comporte de multiples champs concernant notamment la collecte (nom de la station, coordonnées géographiques, date, nom du collecteur, méthode de capture, etc.) et le spécimen lui-même (identifiant unique du lot ou du spécimen, du type MER_I008I0_BRIS_I, famille, genre, espèce, sous-espèce, déterminateur, année de la détermination, nombre de spécimens dans le lot, mode de conservation du spécimen, nom de la plaque de 96 puits dans laquelle est stocké le prélèvement destiné au barcoding, position du prélèvement dans la plaque).

Le barcoding exige clairement des morphologistes un surcroît de travail, qui a pu être partiellement assumé par les molécularistes dans le cas du module marin des expéditions naturalistes, du fait de la présence d'un atelier barcode, mais pas dans l'inventaire de la biodiversité du Mercantour. II peut aussi signifier faire du moins bon travail, comme on l'a vu avec la nécessité de remplacer l'antigel par de l'alcool, à nouveau dans le Mercantour. Dans ce cas, l'absence des molécularistes sur le terrain, liée à l'étalement de l'inventaire dans l'espace et dans le temps, et le recours à certaines méthodes de capture, liée aux types d'animaux collectés (insectes volants), ont accru les exigences des molécularistes et les contraintes pour les morphologistes. On trouve là une partie de l'explication des difficultés plus grandes des molécularistes à enrôler les morphologistes dans le barcoding dans l'inventaire de la biodiversité du Mercantour que dans le module marin des expéditions naturalistes. Cependant, quel que soit le terrain, choisir de contribuer au barcoding affecte les pratiques des systématiciens morphologistes.

Or, tous sont loin d'être convaincus de l'intérêt de la nouvelle technique :

« C'est un peu pénible pour eux, parce que c'est long, hein, à faire. II faut remplir un fichier Excel, mettre les informations... Vu leurs bêtes, ils n'avaient pas envie de faire ça. La plupart n'en voient pas l'intérêt. Ils ne sont pas branchés sur les trucs comme ça » (un morphologiste impliqué dans le volet barcoding de l'inventaire du Mercantour).

II faut dire que le barcoding a suscité de vifs débats dans la communauté académique (voir, par exemple, le numéro ${ }^{6}$ de Trends in Ecology and Evolution paru

6 Volume 18, numéro 2. 
en février 2003). Certains doutent en particulier qu'il soit approprié à leur taxon ${ }^{7}$ de prédilection ou, comme ce morphologiste amateur impliqué dans l'inventaire de la biodiversité du Mercantour, discutent ses fondements théoriques :

« Le barcoding repose sur un certain nombre de... présupposés. Par exemple, un des présupposés c'est que la vitesse de l'évolution, la vitesse des mutations des séquences d'ADN se fait à un rythme constant dans les temps géologiques. C'est une hypothèse qui a le mérite d'être la plus simple mais on n'a aucune évidence que ce soit vrai. Donc, je veux dire, faire de la cladistique $^{8}$ à partir des seules données de génétique moléculaire, ça peut donner des résultats complètement aberrants. D'autant plus qu'en aucun cas on ne séquence tout le génome. D'ailleurs, on tomberait très vite dans des problèmes de variabilité infra-spécifiques ou individuels. »

Plusieurs morphologistes ont en outre estimé qu'une partie excessive du budget des inventaires revenait au barcoding, au détriment du financement du travail de collecte et de tri des spécimens. Une autre réticence concerne la constitution de bases de données susceptibles d'aider des concurrents à identifier des espèces et à résoudre des énigmes taxonomiques, en particulier dans les taxons où la compétition pour la découverte de nouvelles espèces est vive. Pour toutes ces raisons, l'indispensable collaboration des morphologistes au barcoding n'est pas acquise d'emblée. Les molécularistes doivent alors s'employer à l'obtenir en déployant une stratégie d'enrôlement.

\section{UNE STRATÉGIE D'ENRÔLEMENT PLURIELLE}

Le barcoding est une technique récente qui s'est largement diffusée mais qui ne s'est pas (encore ?) totalement imposée. À ce stade, ce sont les molécularistes qui sont demandeurs d'une collaboration et qui déploient des efforts pour enrôler les morphologistes dans le barcoding. Leur stratégie d'enrôlement combine trois composantes qui reposent sur des compétences distinctes. Premièrement, les molécularistes expliquent ce qu'est le barcoding et montrent les pratiques que requiert sa bonne réalisation. Deuxièmement, ils développent une rhétorique propre à rassurer les morphologistes et à les persuader que le barcoding sert l'intérêt général de la discipline. Troisièmement, ils apprennent à différencier les morphologistes en fonction de leur degré d'ouverture au barcoding, cultivant les relations avec les morphologistes favorables ou du moins ouverts au barcoding et évitant les « barcodo-sceptiques ».

7 Un taxon est un ensemble d'individus présentant des caractéristiques communes, comme une espèce ou un groupe d'espèces.

8 La cladistique cherche à établir les relations de parenté entre les taxons. 


\section{Expliquer et montrer}

À partir de 2004, les expéditions naturalistes organisées par le Muséum et Pro-Natura International ont toutes inclus un atelier barcode. L'irruption de la biologie moléculaire au cœur même des collectes naturalistes de terrain s'est faite de manière concomitante avec la participation du MNHN au Consortium for the Barcode of Life évoqué en introduction. Ces enjeux et logiques institutionnels ont certainement joué un rôle dans la décision de l'organisateur de ces expéditions, Philippe, professeur au MNHN, de créer un atelier barcode, et d'embarquer plusieurs molécularistes dont Hervé, professeur de biologie évolutive à Paris, directeur de l'UMR Systématique, Adaptation, Évolution, théoricien peu habitué aux missions naturalistes de terrain et fervent défenseur d'un rapprochement entre approches morphologistes et molécularistes. Dans le module marin de ces expéditions naturalistes, les molécularistes ont dès lors dû former les morphologistes à la préparation des spécimens pour le barcoding. Apprendre à travailler ensemble ne s'est pas fait sans heurts et nous avons déjà indiqué la nette évolution des relations entre l'expédition Santo et l'expédition Atimo Vatae, à Madagascar. Là, nous avons pu filmer une scène de préparation des spécimens en vue du barcoding, qui témoigne de la prise en charge par les molécularistes de véritables séances de formation en direct, tenant compte des spécificités des collecteurs et de leurs conditions de travail.

Solange, une marchande malgache de coquillages, accompagnée de son ami italien, Luigi, marchand lui-même et collectionneur de coquillages du sud malgache, arrivent un matin à l'hôtel de Fort Dauphin où le module marin a installé son laboratoire de plein air. Elle tient dans la main un superbe coquillage vivant et intact, un Turridae, qu'elle offre à Philippe. Après les remerciements d'usage, Philippe vient dire à Nicolas que les villageois ont été invités à rapporter des coquillages vivants. Convaincu qu'il y en aura beaucoup après leur départ de Madagascar, il demande à Nicolas de montrer à Solange comment conserver le tissu et la coquille pour le barcoding, afin qu'elle envoie le matériel à Paris. Nicolas se rend donc avec Solange et Luigi dans le laboratoire. II lui faudra très précisément huit minutes pour leur expliquer comment préparer un mollusque pour le barcoder :

Nicolas : Alors pour les Terebridae, il y a deux solutions, le minimum serait d'enlever l'opercule; en étant rapide on enlève l'opercule, ce qui fait qu'il ne va pas pouvoir se fermer hermétiquement et l'alcool va pénétrer dans le tissu. Le problème si l'opercule reste, c'est qu'il est complètement imperméable à l'alcool, l'alcool ne rentrera pas et l'animal va pourrir. Donc enlever l'opercule. Après, même s'il se rétracte, l'alcool devrait pouvoir rentrer, d'accord ? Ça c'est la première solution. La deuxième solution, si vous avez par exemple une dizaine de spécimens collectés au même endroit, même espèce, vous en prenez cinq, vous les cassez bien net au milieu et cinq intacts, tac bien net, pas en mille morceaux mais en deux morceaux, et les cinq autres intacts, comme ça on aura cinq spécimens parfaits et les cinq autres, si on peut les avoir cassés au milieu mais au moins on aura des coquilles intactes, c'est les deux façons les plus simples 
de faire pour les Terebridae je pense, parce que je vais pas vous demander de les percer, c'est déjà un peu plus compliqué, là on peut essayer.

On voit ici que la préparation des spécimens en vue de leur barcoding ultérieur est devenue une pratique de terrain comme une autre, qui peut être enseignée à des marchands de coquillages sans formation scientifique. En 2006, pendant l'expédition Santo, le barcoding était une technologie controversée, objet de discussions, de conversations, de critiques ou, au contraire, de vifs soutiens. En quatre ans, il est devenu une technologie routinière, que Nicolas le barcodeur est chargé de transmettre aux marchands, collecteurs et amateurs, malgaches et italiens.

Un autre extrait de la même conversation suggère que l'opération d'étiquetage et de codage des fragments de tissu prélevés est ici prise en charge par les barcodeurs :

Le professeur de biologie: tu vas le faire?

Nicolas : je vais m'empresser de le faire ! (suivi de Luigi, Solange et le professeur de biologie, il s'installe à sa table de l'atelier barcode). Nous ici on rajoute un petit label avec un numéro. Là c'est simple, normalement il ne devrait pas s'enfuir.

Le professeur de biologie : tu coupes n'importe quelle partie [du mollusque] ?

Nicolas : le pied, plutôt le pied, voilà tac, je le laisse repartir maintenant, et là ça me suffit comme morceau de tissu, largement. Alors oui un morceau du pied parce que si on coupe les branchies, si on coupe l'estomac, on va être contaminé par des bactéries, par ce qu'il a mangé, etc. Dans le pied, il n'y a que du pied ; quand on peut on prend du pied, en plus si c'est des spécimens gros comme ça c'est facile. Si vous avez des petits spécimens, ne vous embêtez pas : vous percez juste un trou, direct dans l'alcool, parce que sortir des petites bestioles, avec une loupe ça va mais sinon vous allez y passer des heures, voilà maintenant je mets ça dans le tube... je mets de l'alcool, tac... j'ai même un trop gros morceau de tissu, j'en enlèverai un peu et ça [le coquillage] je le mets dans l'alcool aussi dans un sachet avec le tube, comme ça je sais que ce morceau de tissu va avec la bestiole, voilà. »

Dans l'expédition Santo, les spécimens destinés au barcode étaient mis dans de petits tubes ayant eux-mêmes un code-barre. Puis une personne de l'atelier barcode, un biochimiste américain spécialiste du venin des cônes, passait ces codes-barres au laser pour les saisir dans une base de données. Sous Excel, un certain nombre d'informations étaient alors rentrées, colonne par colonne, pour chaque spécimen : la station de prélèvement, le nom de la famille, le nom du genre, le nom de l'espèce lorsqu'il était connu, la présence éventuelle de parasites, et, dans une dernière colonne, quelques précisions sur le nombre de spécimens et le nombre de vouchers ${ }^{9}$, etc.

L'organisation de l'inventaire de la biodiversité du Mercantour ne permettant pas aux molécularistes de venir sur le terrain, il a fallu confier aux morphologistes

9 Spécimen de référence à partir duquel on a obtenu la séquence d'ADN. 
le prélèvement et le conditionnement des parties de spécimens à barcoder. Les explications sur les procédures à suivre ont été délivrées par écrit, par l'intermédiaire d'un « tutoriel » trilingue (anglais, français, italien). Rédigé par le responsable du volet barcoding, au service de systématique moléculaire du Muséum, le tutoriel indique :

« Les prélèvements sont à la charge des taxonomistes ${ }^{10}$. Chaque spécimen devra être prélevé. Selon la taille des spécimens, les individus seront prélevés d'une à cinq pattes. Pour les spécimens trop petits pour être prélevés et pour lesquels l'extraction de l'ADN se fera à partir des individus entiers, il sera nécessaire de les photographier, du moins le premier d'une population barcodée. Dans le but de faciliter le travail moléculaire, les prélèvements doivent être conditionnés par plaque de 96 puits, homogènes dans la mesure du possible (taxon, type de prélèvement, taille...). Il sera demandé de remplir entièrement les plaques, mais si cela n'était pas possible, les plaques non complètes pourront être acceptées si cela est justifié. »

Le responsable du volet barcoding s'est également efforcé d'expliquer comment renseigner les différents champs du tableau Excel à fournir, de la manière la plus claire et la moins rébarbative possible.

Les molécularistes ne doivent pas seulement expliquer ce qu'est le barcoding et montrer comment collecter, préparer et équiper les spécimens pour le rendre possible. Ils doivent aussi persuader qu'il bénéficie à l'ensemble des systématiciens et rassurer les morphologistes quant aux effets du barcoding sur leur pratique et leur statut.

\section{Persuader et rassurer}

Les molécularistes justifient les efforts demandés aux morphologistes par l'apport du barcoding à la systématique et les succès qu'il a permis de remporter. Ces succès sont d'ordre scientifique. Les molécularistes mettent en avant le fait que le barcoding a permis de débrouiller de vieilles énigmes de la discipline et pointent les limites du recours à la seule observation morphologique :

«Dans la majorité des cas, les identifications faites sur le terrain ont été au moins validées, voire précisées. Et dans certains cas où, sur le terrain, on reconnaissait morphologiquement une seule espèce, on s'est aperçu, grâce au barcode qu'il y avait quand même des divergences assez importantes au sein d'un lot de spécimens, qui n'étaient pas aussi marquées sur la morphologie, mais qui étaient quand même présentes, donc sur le moléculaire. Mais après ré-étude des spécimens, effectivement, les spécialistes du groupe ont confirmé que cette espèce était probablement un complexe d'espèces et qu'il y avait sûrement, en effet, plusieurs espèces

10 Les termes taxonomie et systématique sont souvent employés l'un pour l'autre. Nous avons utilisé le terme de systématique dans le texte mais maintenu dans les extraits le terme de taxonomiste lorsqu'il était employé par nos interlocuteurs (avec le sens de systématicien morphologiste dans les propos des molécularistes). 
et le taxonomiste en question, à partir d'observation des photos, a pu effectivement distinguer certaines différences morphologiques que lui seul a vues » (responsable du volet barcoding de l'inventaire du Mercantour).

Pour les molécularistes, il y a donc une supériorité de l'analyse moléculaire sur l'observation des caractères morphologiques, que les morphologistes auraient du mal à admettre : « beaucoup de morphologistes n'acceptent pas, entre guillemets, que l'ADN a raison et que la coquille a tort ».

Comme l'explique Nicolas, les molécularistes ont par ailleurs la possibilité de publier leurs travaux dans des revues internationales de biologie moléculaire, nettement mieux cotées que les revues de systématique morphologique :

« Le fait de mettre du moléculaire te donne accès à des impact factors plus importants, ça c'est évident. La même étude avec la même qualité en termes de délimitation d'espèces avec que du morpho ou avec du morpho et du moléculaire, tu multiplies par deux voire par trois les impact factors, je ne sais pas si c'est bien ou pas bien mais c'est évident que ça, ça a changé. »

Les molécularistes remportent également des succès financiers. Par exemple, le laboratoire de malacologie du Muséum a obtenu d'importants financements d'une fondation privée pour participer à un projet de barcoding de la biodiversité marine. Ils font valoir que ces apports financiers rejaillissent sur l'ensemble de la discipline et que les morphologistes devraient s'en réjouir :

« Nicolas : Plus généralement le barcoding a quand même été un moteur, c'est aussi pour ça que les taxonomistes traditionnels avaient peur du barcoding, beaucoup en ont toujours peur, c'est que ça attire énormément d'argent et ils avaient peur que du coup ça leur prenne l'argent, le peu d'argent qu'on leur donnait pour faire de la taxonomie, ce qu'on remarque plus ou moins, au moins ici en tout cas, c'est que l'argent qu'on récupère avec le barcoding et c'est vrai qu'on récupère plus d'argent avec le mot clé barcoding qu'avec le mot clé morphologie, cet argent-là ils en profitent aussi via justement les projets ; moi quand je développe leur projet de délimitation d'espèces avec du moléculaire, j'ai besoin d'un taxonomiste qui va être capable de me mettre des noms sur les espèces et de faire de la morphologie, ou quand on construit une base de données de référence pour le barcoding au sens strict, on a besoin de quelqu'un pour mettre un nom sur le voucher, là les morphologistes sont toujours indispensables. ॥

En mettant en avant les succès du barcoding, les molécularistes peuvent espérer persuader les morphologistes de collaborer à la diffusion d'une technique présentée comme capable d'améliorer le sort d'une discipline depuis longtemps préoccupée par son statut (Tancoigne, 20ll). Mais il leur faut manier l'argument des succès du barcoding et de sa supposée supériorité avec beaucoup de doigté et de diplomatie. En effet, nombre de morphologistes ont vu et continuent pour certains de voir le barcoding comme une menace pour leur propre pratique. Le statut d'arbitre du gène COI (Waterton, 2010) et la 
prééminence des molécularistes sont vécus par certains comme une dilution voire une confiscation de leur capacité d'expertise, au profit d'un collectif dont le morphologiste n'est plus qu'un membre parmi d'autres, et presque secondaire. Ce sentiment peut conduire au rejet d'une technique perçue comme reléguant les morphologistes à un rang subalterne.

Les craintes des morphologistes sont notamment venues des premiers articles consacrés au barcoding, publiés au début des années 2000 par Paul Hebert et ses collaborateurs (Hebert et al., 2003a, 2003b). Ces articles annonçaient une révolution de la systématique par le barcoding, présenté comme capable non seulement d'assigner à chaque espèce animale une séquence d'ADN caractéristique mais aussi d'identifier de nouvelles espèces et de délimiter les espèces :

«Once established, this microgenomic identification system will overcome the deficits of morphological approaches to species discrimination : the bounds of intraspecific diversity will be quantifiable, sibling species will be recognizable, taxonomic decisions will be objective and all life stages will be recognizable » (Hebert et al., 2003a : 320).

Ces premiers articles ont lancé le coup d'envoi du barcoding. Mais, en suscitant l'inquiétude et les critiques des morphologistes, leur ambition affichée constitue pour les molécularistes un handicap qu'ils tentent de surmonter en révisant à la baisse les promesses initiales du barcoding, ramenées à une forme de marketing scientifique :

« Hebert s'est un peu enflammé ! C'est-à-dire qu'il a voulu que la mayonnaise prenne; il a bien joué son coup! Le problème c'est que dès le premier article, il dit effectivement que le barcoding va aussi permettre de découvrir de nouvelles espèces, parce qu'il voulait vendre son truc quoi et là il a peut-être un petit peu manqué de..., ce n'était peut-être pas assez carré. Et alors ce qui est un peu difficile à saisir c'est que le barcoding permet de découvrir de nouvelles espèces dans le sens où il permet de mettre le doigt dessus, par contre le processus ne s'arrête pas là, il va falloir analyser d'autres caractères pour réellement démontrer que c'est une nouvelle espèce et la délimiter correctement. »

Les molécularistes tiennent par ailleurs des propos rassurants sur le caractère indispensable des connaissances et des compétences des morphologistes. Ils soulignent l'absence de concurrence entre molécularistes et morphologistes et la capacité du barcoding à fédérer les forces, avec l'émergence d'une systématique intégrative combinant analyses moléculaires et observations morphologiques :

« Le travail moléculaire, eux, ils voient ça un peu comme quelque chose qui va leur donner le coup de grâce, alors que non, c'est un travail qui est complémentaire. La valeur du barcode repose sur le travail d'un taxonomiste initialement, parce que nous, on travaille sur les spécimens identifiés par les taxonomistes. Nous, on ne prétend pas identifier les 
spécimens, on n'a pas la compétence. Nous, on caractérise par l'ADN les espèces définies par les taxonomistes. Mais en aucun cas, on ne veut s'affranchir des taxonomistes " (responsable du volet barcoding de l'inventaire du Mercantour).

Les molécularistes doivent en définitive trouver un équilibre difficile à tenir entre bien circonscrire la plus-value d'une nouvelle technique pour éviter des critiques scientifiquement fondées et des réactions de rejet de la part des morphologistes et mettre en avant les apports du barcoding à la systématique, qui justifient que les morphologistes prennent en compte leurs demandes. II en résulte une certaine tension dans les discours, qui transparaît par exemple dans la distinction entre de bons usages du label barcoding (la construction d'une base de données globale qui associe à chaque espèce identifiée une séquence d'ADN unique) et des usages qualifiés d'abusifs mais largement répandus, qui associent le barcoding à l'identification d'espèces nouvelles ou à la délimitation d'espèces. Cette tension transparaît aussi dans des formules concessives. Les barcodeurs se montrent attentifs à ne pas se positionner en détenteurs d'une vérité absolue. Ainsi, après avoir affirmé que « l'ADN a raison et la coquille a tort », l'un d'eux a aussitôt ajouté : « mais il y a aussi des cas où la coquille a raison $॥ "$.

Les molécularistes développent au final une rhétorique assez subtile qui chante les mérites du barcoding en tentant de ménager les savoirs et savoirfaire des morphologistes. La stratégie d'enrôlement des molécularistes repose encore sur leur capacité à identifier les morphologistes les plus réceptifs à la nouvelle technique.

\section{Différencier les morphologistes}

Les systématiciens qui participent aux grands inventaires de la biodiversité tels que ceux considérés ici ont tous un point commun : ils sont considérés comme faisant partie des meilleurs spécialistes de leurs taxons. Mais ils sont par ailleurs très différents les uns des autres. On trouve parmi eux des professionnels et des amateurs ${ }^{12}$; certains travaillent en équipe et d'autres en binôme ou en solitaire ; les milieux naturels qu'ils fréquentent, leurs méthodes d'investigation et de collecte et les taxons auxquels ils s'intéressent sont d'une surprenante variété. Ils constituent ainsi un monde social des plus hétérogènes. Beaucoup ont par ailleurs des personnalités très originales et indépendantes. Pour ne prendre qu'un exemple, un systématicien, rencontré dans le Mercantour, a effectué toute sa carrière au CNRS en publiant en français plutôt qu'en anglais

II Cependant, leurs propos témoignent parfois d'une forme de condescendance et de sentiment de supériorité. Ils qualifient par exemple volontiers les morphologistes de « systématiciens traditionnels $॥$.

12 Les participants à l'inventaire de la biodiversité du Mercantour étaient pour deux tiers environ des professionnels et pour un tiers des amateurs. 
et en œuvrant pour le développement de l'esperanto, au nom de la démocratie linguistique. Face à cette diversité et au caractère souvent bien trempé de leurs interlocuteurs, les barcodeurs s'efforcent d'ajuster les dispositifs d'enrôlement à la singularité des situations et des interactions. Le responsable du volet barcoding de l'inventaire du Mercantour a ainsi rencontré, à Paris, plusieurs morphologistes dans l'espoir de les motiver à contribuer au barcoding en envoyant des spécimens au service de systématique moléculaire. Ces rencontres ont facilité le développement d'approches personnalisées du barcoding et un enrôlement individualisé et en douceur dans une démarche standardisée :

« Ça a été assez compliqué au niveau des taxonomistes de mettre en place des choses qui ne leur mettent pas des bâtons dans les roues. II faut qu'ils voient le travail qu'on doit faire ensemble plutôt comme un bénéfice pour eux que comme une contrainte. Et donc ça, ça n'a pas toujours été évident de présenter les choses de manière à ce qu'ils se sentent aidés plutôt que contraints. Donc il y a eu pas mal de petites réunions informelles, pour se retrouver, discuter sur certains points, voir comment faciliter, comment imposer le moins possible aux taxonomistes... Donc c'est pas mal de prises de contact direct, de manière informelle. C'étaient des coups de téléphone : "Est-ce que je peux passer te voir ?" "Voilà, OK". Ou un petit mail : "OK, je passe la semaine prochaine" (un membre du service de systématique moléculaire).

Les molécularistes s'efforcent par ailleurs de repérer des morphologistes intéressés par le barcoding, auxquels ils s'adressent en particulier :

« Isabelle : Et pourquoi vous avez choisi d'aller voir ces gens-là ? Parce qu'ils sont responsables de collection ?

Le responsable du volet barcoding de l'inventaire du Mercantour: Non, pas forcément. C'est des gens avec qui j'ai eu l'occasion de prendre contact relativement facilement, des gens avec qui le contact est bien passé, et des gens qui étaient volontaires, en fait. Parce que là, on était vraiment, pour le moment, dans une phase de mise au point. Donc je n'ai pas pris contact avec tout le monde. J'ai pris contact avec ceux qui étaient là, ceux qui étaient motivés et ceux qui étaient intéressés. Notamment Q., c'est quelqu'un qui est intéressé, qui a du matériel qui est prêt, qui a du temps, un peu, à consacrer à ça et qui est intéressé par avoir des séquences sur ses bêtes et... Voilà. Donc lui, en l'occurrence, il m'a servi de taxonomiste test ! »

Au contraire, les deux extraits suivants suggèrent que les molécularistes tendent à éviter les morphologistes critiques ou peu intéressés par la nouvelle technique :

« Isabelle : Il y a d'autres noms qui me viennent à l'esprit, de personnes qui me paraissent être pas mal impliquées dans l'inventaire. Je pense à C. ou [/] Le responsable du volet barcoding de l'inventaire du Mercantour : Alors C., c'est pareil, on est allé la voir. Et d'ailleurs, on est allé la voir dans la semaine. Mais C. est débordée, C. n’a pas le temps, C.... C'est assez compliqué. Je n'ai pas insisté. » 
« Nicolas : J'ai un collègue [au Muséum], chaque fois que je lui amenais un résultat qui ne concordait pas avec la morpho[logie], il me disait que je me trompais! Non non tu as faux! Alors tu sais quand tu commences ta thèse, je le croyais, et puis limite je refaisais ma séquence trois fois et je trouvais toujours la même, je ne savais plus quoi faire parce que je savais que je n'avais pas fait d'erreur, mais il ne me croyait pas, il ne me croyait pas du tout, et puis entre temps ça a été corroboré par un collègue de façon indépendante, et finalement j'avais raison mais finalement après deux ans ou trois ans il l'a accepté.

Elsa : Et là maintenant il est plus facile à convaincre ou pas ?

Nicolas : Non, pas forcément, il n'est pas très ouvert. »

Les morphologistes ne sont pas tous soit ouverts soit fermés au barcoding. Certains peuvent poser des conditions à leur collaboration, que les molécularistes doivent également repérer et peser. C'est le cas des marchands de coquillages comme Luigi et Solange (cf. supra). Luigi conclut la discussion précédemment rapportée en précisant que le Terebridae apporté par Solange était un cadeau, mais qu'un contrat devra être signé pour que d'autres spécimens soient fournis pour le barcoding. Un tel exemple de rétribution financière d'une collaboration au barcoding est rare, peut-être même exceptionnel ; nous n'en avons pas observé dans le cas de l'inventaire du Mercantour. Mais il éclaire de manière intéressante les pratiques d'enrôlement dans un nouveau champ techno-scientifique. Pour enrôler Luigi et Solange dans le barcoding, les molécularistes doivent leur montrer comment prélever et conserver les tissus de manière à conserver l'ADN, opérations qui ne semblent pas les avoir rebutés ni effrayés et qui a retenu toute leur attention. Ils doivent aussi prendre en compte l'identité sociale de ces marchands de coquillages. À cette condition, Luigi et Solange semblent disposés à assumer une nouvelle mission pour les malacologues professionnels : préparer pour le barcoding les spécimens de coquillages qu'ils achètent dans le Sud de Madagascar à leur réseau de pêcheurs, avant de les envoyer ou de les donner en mains propres aux malacologistes du Muséum de Paris.

Les molécularistes effectuent donc un travail fin de différenciation du premier public que constituent les systématiciens morphologistes : ils doivent apprendre à repérer ceux qui sont prêts à collaborer, trouver les arguments qui font mouche et éventuellement répondre favorablement aux conditions posées par leurs interlocuteurs. Ce travail contraste fortement avec l'opération d'homogénéisation du public général décrite par Ellis, Waterton et Wynne (cf. supra). Le succès du barcoding repose ainsi à la fois sur l'aptitude de ses promoteurs à produire un public général indifférencié et sur la capacité de ses praticiens à identifier dans le premier public des systématiciens les personnes les mieux disposées envers une technique qui modifie leurs pratiques.

En définitive, les molécularistes mobilisent un ensemble de compétences, pédagogiques (expliquer et montrer), rhétoriques (persuader et rassurer) et sociales (différencier les morphologistes et personnaliser les approches) pour tenter d'enrôler les morphologistes dans le barcoding et initier une collaboration indispensable à leur entreprise mais délicate. Ces façons d'enrôler se retrouvent 
dans nos différents terrains d'enquête mais la comparaison entre le module marin des expéditions marines et l'inventaire généralisé de la biodiversité du Mercantour permet de mettre au jour un certain nombre de circonstances qui les favorisent ou les compliquent.

\section{DES CIRCONSTANCES (DÉ)FAVORABLES}

On l'a dit, l'enrôlement des morphologistes dans le barcoding a dans l'ensemble été plus facile et plus fructueux dans le module marin des expéditions naturalistes que dans l'inventaire de la biodiversité du Mercantour, alors que les efforts déployés dans les deux cas par les molécularistes apparaissent d'intensité similaire. Deux circonstances semblent avoir favorisé la stratégie d'enrôlement des morphologistes par les molécularistes dans le cas des modules marins : la participation physique des molécularistes aux expéditions ; la présence d'un morphologiste converti au barcoding à la tête de ces dernières.

\section{La possibilité de se frotter les uns aux autres}

La présence physique des barcodeurs dans le module marin des expéditions naturalistes a joué un rôle important dans l'enrôlement des systématiciens de terrain. En effet, ils ont pu faire des démonstrations concrètes des techniques de prélèvement et de conditionnement requises par le barcoding, alors qu'ils ont dû recourir à des explications écrites dans l'inventaire de la biodiversité du Mercantour. La remise d'un tutoriel, même rédigé dans l'objectif d'être aussi accessible que possible, ne remplace pas une séance de travaux pratiques. Par ailleurs, la participation des molécularistes aux modules marins leur a permis d'assumer une partie de la surcharge de travail induite par le barcoding. Ce sont eux, en effet, qui ont réalisé une partie des prélèvements ainsi que la saisie des données. Dans le Mercantour aussi, le responsable du barcoding s'est efforcé de décharger les morphologistes des contraintes du barcoding mais il n'a pas pu intervenir à la même hauteur que les molécularistes impliqués dans les modules marins, du fait de son éloignement du terrain :

« Le responsable du volet barcoding de l'inventaire du Mercantour : Mais pour le moment, il n'y a pas une très, très forte participation des taxonomistes.

Isabelle : Oui, ça commence.

Le responsable du volet barcoding de l'inventaire du Mercantour : moi, je prends contact avec eux et j'essaie de les motiver et dans certains cas, je fais des prélèvements moi-même, enfin, je fais les choses moi-même pour... pour faire avancer, voilà, pour avancer le travail ». 
Au-delà de la possibilité de montrer comment préparer et équiper les spécimens et de faire une partie au moins du travail, la présence des molécularistes sur le terrain a permis aux systématiciens de se côtoyer. Logés dans les mêmes bâtiments et travaillant à proximité les uns des autres voire ensemble, les molécularistes et les morphologistes impliqués dans les modules marins ont eu toute la durée des expéditions, soit plusieurs semaines, pour se connaître, échanger et confronter leurs points de vue, de manière plutôt conflictuelle à Santo et plus apaisée ensuite. Ces interactions ont été plus rares et limitées dans le cas de l'inventaire de la biodiversité du Mercantour, les molécularistes n'ayant pas participé au travail de terrain :

« Je ne suis pas allé dans le Parc à proprement parler pour... Moi, je ne suis jamais allé récolter, je ne suis jamais allé en mission. Après, on a des réunions, le Parc organise des réunions assez régulièrement pour savoir où ça en est » (responsable du volet barcoding de l'inventaire du Mercantour).

Une autre différence importante entre nos terrains d'enquête concerne l'implication de convertis charismatiques.

\section{L'implication de convertis charismatiques}

L'un des principaux leaders des expéditions naturalistes dans lesquelles s'insèrent les modules marins est un morphologiste qui s'est d'abord montré très sceptique envers le barcoding avant d'en devenir un fervent défenseur :

« Nicolas : Philippe est l'exemple inverse : en 2002, 2003, l'ADN c'était limite quèsaco et je ne veux pas en entendre parler et puis maintenant il engueule les taxonomistes qui publient sans faire de moléculaire. II y a eu chez lui un changement radical! Moi je ne l'ai pas connu avant mais tout le monde dit que c'est hallucinant le changement qu'il y a eu chez Philippe ! Lui pour le coup, le barcoding a changé sa façon de travailler, complètement, complètement. »

Cette « conversion " spectaculaire au barcoding semble avoir été liée au constat que la nouvelle technique a permis de démêler des problèmes taxonomiques longtemps demeurés insolubles:

«Nicolas: Ce qui l'a vraiment convaincu, c'est les Conoidea; pendant quarante ans il ne savait pas quoi en faire. Les taxonomistes se contredisaient tout le temps, ça faisait un siècle qu'ils essayaient de proposer une classification des Conoidea et en cinq ans de moléculaire sur le groupe on a proposé une classification des Conoidea qui n'a jamais été aussi stable. Je ne promets pas qu'elle ne va pas changer mais elle sera beaucoup plus stable que ce qu'il y avait avant. $"$ 
Lui-même met en avant les découvertes de nouvelles espèces permises par le barcoding lors des expéditions :

«Grâce au moléculaire, sur le matériel collecté à Santo, on a décrit deux espèces nouvelles dont l'une était presque indiscernable morphologiquement. C'est incroyablement porteur ! Je ne me rappelle pas si je tenais déjà le même discours au moment de Santo mais la démocratisation du moléculaire en termes de facilité, de coût de séquençage, facilité de conservation des tissus, ça a complètement changé effectivement les relations entre les expéditions et le moléculaire. Et puis au point de vue scientifique, c'est révolutionnaire ! C'est vraiment révolutionnaire ! »

Quoi qu'il en soit, en 2010, cette nouvelle technologie était devenue à ses yeux indispensable à toute exploration et collecte naturalistes. Or ce converti au barcoding dispose d'une grande autorité auprès des morphologistes, de fait de ses nombreuses publications académiques et de la visibilité qu'il a su donner à la discipline, en relançant de grands inventaires de la biodiversité :

« Nicolas : Philippe fait un peu acte d'autorité. Quand l'amateur arrive et que Philippe lui dit non non c'est l'ADN qui a raison, l'amateur il dit ok, tu as raison! II ne va pas le contredire. ॥

Ses réprimandes envers les critiques et les sceptiques visent aussi des morphologistes professionnels et peuvent être interprétées comme des formes de sanction morale des morphologistes récalcitrants ${ }^{13}$.

La situation a été très différente dans l'inventaire de la biodiversité du Mercantour, essentiellement porté et organisé par le parc national du Mercantour. L'organisation de l'inventaire a été confiée à une jeune chargée de mission extrêmement active, titulaire d'un master en écologie, qui a œuvré pour la mise en place d'un volet barcoding. Plusieurs années après le démarrage de l'inventaire, un étudiant de master a été recruté dans le cadre d'un CDD au service de systématique moléculaire pour prendre en charge le volet barcode de l'inventaire. Ces deux personnes n'ont pas ménagé leur peine pour inciter les morphologistes à contribuer au barcoding. Mais leur statut et le fait qu'ils ne font partie de la communauté des systématiciens morphologistes ne leur ont pas permis d'exercer la même autorité sur les morphologistes que le leader des expéditions naturalistes.

Tout de même, il y a bien eu, dans l'inventaire de la biodiversité du Mercantour aussi, des morphologistes réputés que le barcoding intéresse et qui jouent un rôle important dans sa diffusion, en relayant auprès de leurs pairs les arguments des molécularistes:

13 C'est d'ailleurs là le seul exemple de sanction que nous ayons rencontré dans nos différents terrains, alors que les projets collaboratifs punissent parfois, d'une manière ou d'une autre, ceux de leurs membres jugés mauvais collaborateurs (Harris, à paraître). Mais le barcoding est sans doute une technologie trop récente pour que ses promoteurs puissent se permettre de recourir à des sanctions contre les morphologistes récalcitrants. 
« Et alors un des intérêts, justement, de faire ce travail collectif, c'est de leur montrer qu'on peut aller plus loin à certains niveaux, on peut débrouiller certains problèmes qui traînent depuis des années, des dizaines d'années. On peut contribuer à résoudre les problèmes avec le barcode. Il y en a qui commencent à s'y intéresser, qui commencent disons à réagir positivement » (un morphologiste impliqué dans le volet barcoding de l'inventaire du Mercantour).

\section{CONCLUSION}

Avec le barcoding, les systématiciens molécularistes ont inventé et promu une nouvelle technique d'investigation du vivant. Cette technique requiert la contribution active des systématiciens morphologistes, qui ont une autre manière de connaître le vivant et ne sont pas d'emblée acquis à cette technique. Loin de dépendre uniquement d'aspects techniques et scientifiques, le succès du barcoding repose donc aussi sur la capacité des molécularistes à enrôler les morphologistes et à mettre en œuvre des compétences pédagogiques, rhétoriques et sociales.

Nos enquêtes ont mis en évidence la stratégie d'enrôlement plurielle adoptée par les molécularistes : ils expliquent ce qu'est le barcoding et montrent comment collecter, préparer et équiper les spécimens; ils cherchent à persuader les morphologistes de l'intérêt de la nouvelle technique pour l'ensemble de la systématique et à apaiser leurs craintes de disparaître ou d'être dominés ; ils apprennent à repérer le degré d'ouverture des morphologistes au barcoding. Les formes de rétribution des morphologistes coopératifs et de sanction des morphologistes récalcitrants sont en revanche peu présentes, ce qui renvoie peut-être à la jeunesse du barcoding.

En adoptant une approche comparative et longitudinale, nous avons par ailleurs pu montrer que les dispositifs d'enrôlement des morphologistes prennent des formes variées selon les terrains : formation en direct des morphologistes et signature de contrats avec des marchands de coquillages dans les modules marins des expéditions naturalistes ; élaboration d'un tutoriel trilingue et prises de contact informelles dans l'inventaire de la biodiversité du Mercantour. La diffusion d'une technologie scientifique standardisée et globalisée passe par des dispositifs d'enrôlement qui sont, eux, socialement et spatialement situés. Notre approche a également permis de mieux comprendre pourquoi le barcoding a plutôt mieux marché dans les modules marins des expéditions naturalistes que dans l'inventaire de la biodiversité du Mercantour alors que, dans les deux cas, les molécularistes ont déployé d'importants efforts en direction des morphologistes. D'une part, le barcoding a occasionné moins de contraintes dans les modules marins que dans le Mercantour, où les molécularistes n'ont pas pu assumer une partie de la surcharge de travail induite par la nouvelle technique et où 
certaines méthodes de capture se sont révélées incompatibles avec le barcoding. D'autre part, la cohabitation des molécularistes et des morphologistes pendant toute la durée des expéditions et la présence d'un morphologiste converti au barcoding à la tête de ces expéditions ont constitué des facteurs très favorables à l'enrôlement des morphologistes dans le cas des modules marins.

En définitive, le succès d'une technologie comme le barcoding apparaît fortement lié à la capacité de ses promoteurs à s'adapter à la très grande diversité et singularité des systématiciens morphologistes qu'il leur faut parvenir à enrôler et des situations d'exercice de leur pratique. Ces systématiciens morphologistes constituent le «premier public » du barcoding. Ellis, Waterton et Wynne ont insisté sur l'opération d'homogénéisation du public général sur laquelle repose le barcoding. Notre enquête nous amène pour notre part à souligner le travail de différenciation du premier public auquel se livrent les molécularistes. Les deux conclusions ne sont pas contradictoires : en même temps qu'ils fabriquent un public général homogène, caractérisé par son bio-illettrisme, son envie d'apprendre à « lire ॥ la biodiversité et sa capacité à être transformé par cette lecture en un public responsable, les promoteurs du barcoding prêtent une grande attention aux différences entre les morphologistes et aux conditions concrètes dans lesquelles ils opèrent et prennent en compte ces différences dans leurs dispositifs d'enrôlement. Les molécularistes homogénéisent donc le public général du barcoding mais pas son premier public. Étant donné la diversité du vivant, ils ont bien tout intérêt à ce que se maintienne et si possible se développe une diversité de systématiciens morphologistes susceptibles de collaborer à leur entreprise.

\section{Remerciements}

Ce texte est issu de deux projets de recherche financés par l'ANR dans le cadre du programme "Sciences, technologies et savoirs en société ", l'ANR Pan-Bioptique ANR-09-SSOC-053-0I et l'ANR Expébiodiv ANR-09-SSOC-052.

Nous adressons nos remerciements à l'ensemble des personnes enquêtées. Nous tenons également à exprimer notre gratitude à Nicolas,

à Raphaël Larrère et à plusieurs évaluateurs anonymes pour leurs commentaires et suggestions sur une version antérieure de ce texte. Nous restons bien sûr seules responsables des interprétations proposées.

\section{RÉFÉRENCES}

Callon, M. (1986). Éléments pour une sociologie de la traduction. La domestication des coquilles Saint-Jacques et des marins-pêcheurs dans la baie de Saint-Brieuc. Année sociologique, 36, 169-208.

Charvolin, F., Micoud, A., Nyhart, L. K. (2007). Des sciences citoyennes ? La question de l'amateur dans les sciences naturalistes. Paris : L'Aube. 
Dupré, J. (2006). Real but Modest Gains from Genetic Barcoding. Genomics, Society and Policy, 3, 4 |-43.

Ellis, R. (2008). Rethinking the value of biological specimens: laboratories, museums and the Barcoding of Life Initiative. Museum and society, 6, 172-191.

Ellis, R., Waterton, C., Wynne, B. (2009). Taxonomy, biodiversity and their publics in twenty-first-century DNA barcoding. Public Understanding of Science, 19, 497-5I 2.

Faugère, E. (2008). L'exploration contemporaine de la biodiversité. Approche anthropologique de l'expédition Santo 2006. Le journal de la société des océanistes [En ligne]. I26-127, 195-206.

Granjou, C., Mauz, I. (2009). Quand l'identité de l'objet-frontière se construit chemin faisant. Le cas de l'estimation de l'effectif de la population de loups en France. Revue d'Anthropologie des Connaissances, 3 (I), 29-49.

Harris, F. (20/3) à paraître. Transdisciplinary environmental research: building trust across professional cultures. Environmental Science \& Policy.

Heaton, L, Millerand, F., Crespel, E., Proulx, S. (20I I). La réactualisation de la contribution des amateurs à la botanique. Terrains et Travaux, I8,155-I73.

Hebert, P. D. N., Cywinska, A., Ball, S. L., de Waard, J. R., (2003a). Biological identifications through DNA barcodes. Proc. R. Soc. Lond. B, 270, 313-32I.

Hebert, P. D. N., Ratsingham, S., de Waard, J. R. (2003b). Barcoding animal life: cytochrome c oxidase subunit I divergences among closely related species, Proc. R. Soc. Lond. B 270 (Suppl.), S96-S99.

Hollingsworth, P. M. (2007). DNA barcoding: potential users. Genomics, Society and Policy, 3, 44-47.

Kohler, R. (2002). Landscapes and labscapes: exploring the lab-field border in biology. Chicago, IL: University of Chicago Press.

Kohler, R. (2006). All creatures. Naturalists, collectors, and biodiversity, I850-1950. Princeton, Oxford: Princeton University Press.

Larson, B. M. H. (2007). DNA barcoding: the social frontier. Frontiers in Ecology, 5, 437-442. Latour, B. (1989). La science en action. Paris : La Découverte.

Lawrence, A. (2006). No personal motive? Volunteers, biodiversity, and the false dichotomies of participation. Ethics, place and environment, 9, 279-298.

Mauz, I., Granjou, C. (2013). A New Border Zone in Science. Collaboration and tensions between Modelling Ecologists and Field Naturalists. Science as Culture, DOI:10. 1080/0950 5431.2012 .753047

Parker, J., Vermeulen, N., Penders, B. (dir.) (2010). Collaboration in the new life sciences. Ashgate.

Pickstone, J. V. (2000). Ways of knowing. A new history of science, technology and medicine. Manchester: Manchester University Press.

Puillandre, N., Bouchet, P., Boisselier-Dubayle, M.-C., Brisset, J., Buge, B, Castelin, M., Chagnoux, S., Christophe, T., Corbari, L., Lambourdière, J., Lozonet, P., Marani, G., Rivasseau, A., Silva, N., Terryn, Y., Tillier, S., Utgé, J., Samadi, S. (20I2). New taxonomy and old collections: integrating DNA barcoding into the collection curation process. Molecular Ecology Resources, 12, 396-402.

Strasser, B. (20I I). The experimenter's museum: GenBank, natural history, and the moral economies of biomedicine. Isis. 102, 60-96.

Strasser, B., de Chadarevian, S. (20I I). The Comparative and the Exemplary: Revisiting the Early History of molecular Biology. History of Science, 49, 317-336.

Strasser, B. (20I2). Collecting Nature: Practices, Styles, and Narratives, Osiris, 27, I, 303-340.

Tancoigne, E. (20I I). Évaluer la santé de la taxonomie zoologique : histoire, méthodes et enjeux contemporains. Thèse en Systématique et évolution, Muséum National d'Histoire Naturelle, 212 p.

Vinck, D. (20I I) Taking intermediary objects and equipping work into account in the study of engineering practices. Engineering Studies, 3, I, 25-44, First published on II February 20II (iFirst). 
Waterton, C., Ellis, R. (2006). Amateurs as Experts: Harnessing New Networks for Biodiversity. End of Award report, lancaster. CSEC, Lancaster University. http://www. lancs.ac.uk/fass/centres/csec/pubs.htm.

Waterton C. (20I0). Barcoding nature: strategic naturalization as innovatory practice in the genomic ordering of things. The sociological review, 58, I52-I7I.

Isabelle MAUZ est sociologue au centre Irstea de Grenoble. Elle étudie les nouveaux modes d'investigation et de gestion de la nature à l'ère de la biodiversité. Elle s'est intéressée au travail des systématiciens dans le cadre du projet PAN-Bioptique (« Les institutions émergentes de la biodiversité. Inventorier, numériser, expertiser la nature »), coordonné par Céline Granjou et financé par l'Agence Nationale de la Recherche programme «Sciences, technologies et savoirs en société. Enjeux actuels, questions historiques », ANR-09-SSOC-053-0I.

Affiliation Irstea - Centre de Grenoble

2 rue de la Papeterie BP 76

38402 Saint-Martin d'Hères cedex

Courriel_isabelle.mauz@irstea.fr

Elsa FAUGÈRE est ethnologue à l'Inra. Elle a commencé en 2005 à prendre comme sujet d'étude les grandes expéditions naturalistes, en commençant par l'expédition Santo qui s'est déroulée en 2006 au Vanuatu. Elle poursuit ses travaux dans le cadre d'un projet de recherche qu'elle coordonne (2010-2013), intitulé « Expébiodiv. Étude pluridisciplinaire des grandes expéditions naturalistes contemporaines », financé par le même programme de l'ANR que PAN-bioptique.

Affiliation INRA Centre de Recherche PACA

228 route de l'Aérodrome

CS 40509

Domaine Saint Paul - Site Agroparc

84914 Avignon Cedex 9

Courriel_elsa.faugere@avignon.inra.fr

\section{Abstract: THE INTRODUCTION OF BARCODING IN SYSTEMATICS. A STUDY OF SCIENTIFIC ENROLMENT PRACTICES}

Barcoding is a recent technology introduced in an old science, systematics, which aims to identify and classify species. Invented and promoted by one part of the systematicians (the molecularists), its success demands an active collaboration of 
those systematicians who base their work on the observation of morphologic characters (the morphologists). Drawing on empirical surveys carried out in contrasted fields, we analyse how the molecularists seek to enrol the morphologists in barcoding. We show that the molecularists deploy a pluralistic enrolment strategy, based on a set of pedagogical, rhetorical and social competencies. This strategy enables them to take into account the high diversity and particularity of the morphologists and their work conditions. We also underline the factors favouring this enrolment strategy, such as the molecularists' participation in the collection campaigns and the support of morphologists converted to barcoding.

Keywords: enrolment, collaboration, systematics, barcoding, inventory, biodiversity

\section{RESUMEN: LA INTRODUCCIÓN DEL BARCODING EN LA SISTEMÁTICA}

El barcoding es una técnica recientemente introducida en una vieja disciplina científica, sistemática, cuyo objetivo es identificar y clasificar las especies. Inventado y promovido por algunos sistemáticos (los molecularistas), su éxito necesita la colaboración activa de los sistemáticos que basan su trabajo en la observación de los caracteres morfológicos (los morfólogos). Sobre la base de encuestas en terrenos distintos, analizamos cómo los molecularistas se esfuerzan de enrolar los morfólogos en el barcoding. Mostramos que los molecularistas adoptan una estrategia de enrolamiento plural, basada en un conjunto de competencias pedagógicas, retóricas y sociales. Esta estrategia les permite tomar en cuenta la diversidad y singularidad de los morfólogos y de las condiciones para el ejercicio de su práctica. También identificamos los factores que favorecen la estrategia de enrolamiento, como la participación de molecularistas en campañas de recolección y el apoyo de morfólogos convertidos en el barcoding.

Palabras-claves: enrolamiento, collaboraciòn, sistemàtica, barcoding, inventario, biodiversidad 